\title{
Reduced and oxidized glutathione in the substantia nigra of patients with Parkinson's disease
}

\author{
E. Sofic ${ }^{\mathrm{a}}$, K.W. Lange ${ }^{\mathrm{a}}$, K. Jellinger ${ }^{\mathrm{b}}$ and P. Riederer ${ }^{\mathrm{a}}$ \\ "Department of Clinical Neurochemistry, University Hospital for Nervous Diseases, University of Würzburg. Würzburg (FRG) and " Ludwig Boltzmann \\ Institute of Clinical Neurobiology. Lain= Hospital, Vienna (Austria)
}

(Received 18 March 1992; Revised version received 4 May 1992; Accepted 4 May 1992)

Key words: Reduced glutathione; Oxidized glutathione; Substantia nigra; Brain; Human; Parkinson's disease

\begin{abstract}
Reduced and oxidized glutathione concentrations in post-mortem brain tissue from the substantia nigra of control subjects and patients with neuropathologically confirmed Parkinson's disease were measured by a coulometric method using high-pressure liquid chromatography and electrochemical detection. Reduced glutathione concentrations were decreased in the substantia nigra of parkinsonian patients compared with controls. Differences in the concentration of oxidized glutathione and in the percentage of oxidized glutathione of the total glutathione were not observed between parkinsonian and control subjects. The finding that oxidized glutathione is not decreased in Parkinson's disease suggests that the decrease in reduced glutathione is not exclusively the consequence of neuronal loss in the substantia nigra but may indicate a state of oxidative stress.
\end{abstract}

Parkinson's disease is a slowly progressive neurodegenerative disorder which is characterized pathologically by the loss of pigmented neurones from the substantia nigra. The aetiology of this degeneration is unknown. However, endogenous radicals generated by hydrogen peroxide [3], toxic $\alpha, \beta$-unsaturated aldehydes which could react with reduced glutathione (GSH) [13, 19, 23] or metabolic compounds of the catecholamine metabolism such as 5-S-cysteinyldopamine [14] and 6-hydroxydopamine [7] could all potentially trigger Parkinson's disease. Toxic compounds formed in the brain are normally inactivated by various protective mechanisms. These mechanisms, however, may be impaired in Parkinson's disease, e.g. reduced activity of catalase, peroxidase and glutathione peroxidase and diminished concentrations of GSH have been found in the substantia nigra in Parkinson's disease $[1,7,10,12]$. GSH in the substantia nigra and enzymes that utilize GSH for free radical detoxification, such as glutathione peroxidase and glutathione-S-transferase, probably play an important role in protecting dopaminergic nigrostriatal neurones from damage by 1-methyl-4-phenyl-1,2,3,6-tetrahydropyridine (MPTP), or from other MPTP-like neurotoxins which may cause Parkinson's disease in humans. Reduced levels of GSH have been found in the brainstem of

Correspondence: $\mathrm{P}$. Riederer, Klinische Neurochemie, UniversitätsNervenklinik, Füchsleinstr. 15, 8700 Würzburg, FRG. mice following administration of MPTP $[13,23]$. The MPTP-induced depletion of GSH in the brainstem of mice was prevented by pre-treatment with antioxidants [23].

In Parkinson's disease the surviving neurones of the nigrostriatal pathway exhibit an increased dopamine turnover [6]. This could theoretically be associated with oxidative stress as a consequence of increased production of hydrogen peroxide during the oxidative deamination of dopamine by monoamine oxidase (MAO). In the mouse, a reserpine-induced increase in pre-synaptic dopamine turnover caused a rise in the level of oxidized glutathione (glutathione disulfide, GSSG). The formation of GSSG could be blocked by the MAO-A inhibitor clorgyline [18]. Oxidative metabolism of dopamine in the brain is associated with the production of hydrogen peroxide and may be reflected by oxidation of GSH to GSSG [15, 21]. We have therefore examined the concentrations of GSH and GSSG in the substantia nigra of patients with Parkinson's disease and matched control subjects.

Brain tissue from the substantia nigra was obtained at autopsy from four patients with Parkinson's disease and from four age-matched control subjects with no evidence of neurological or psychiatric disease (Table I). The clinical diagnosis was confirmed in all cases by neuropathological examination. Patients with Parkinson's disease had all received L-DOPA combined with the peripheral 
decarboxylase inhibitor benserazide, amantadine sulfate and anticholinergics. The brain tissue was frozen at $-80^{\circ} \mathrm{C}$ until analysis.

GSH and GSSG were measured by a coulometric method using high-pressure liquid chromatography (HPLC) and electrochemical detection (ESA coulochem detector). The effluent was $0.015 \mathrm{M}$ o-phosphoric acid. Compounds were separated on an RP-18 Spherisorb $5 \mu$ $(100 \times 4.6 \mathrm{~mm})$ column. Flow rate was $0.5 \mathrm{ml} / \mathrm{min}$. For electrochemical oxidation the detectors were held at $+0.02 \mathrm{~V}(\mathrm{~T} 1)$ and $+0.30 \mathrm{~V}(\mathrm{~T} 2)$. As reference substance, $10 \mu \mathrm{l}$ of $3.25 \mu \mathrm{M}$ GSH (Sigma, St. Louis, MO, USA) in $0.015 \mathrm{M}$ o-phosphoric acid were used. GSSG was reduced at $\mathrm{pH} 7.5$ by $\beta$-nicotinamide-dinucleotide phosphate and glutathione reductase (EC 1.6.4.2.) to regenerate GSH. Brain tissue was homogenized in $0.015 \mathrm{M}$ ophosphoric acid and centrifuged at $15,000 \mathrm{~g}$ for $10 \mathrm{~min}$ at $4^{\circ} \mathrm{C}$. The supernatants were used for HPLC injections [17]. All results were analyzed using Wilcoxon's ranksum test [22].

The concentration of GSH was reduced in the substantia nigra of patients with Parkinson's disease compared with control subjects (Table I). Differences in the GSSG concentration and in the percentage of GSSG of the total glutathione between parkinsonian and control subjects were not observed.

The present study has investigated GSH and GSSG concentrations in the substantia nigra in normal controls and in Parkinson`s disease. The GSH and GSSG concentrations measured in control subjects are well in accord with the results of previous studies [16]. Levels of GSSG were about $2.3 \%$ of the total glutathione. These results conflict with those indicating that about $90 \%$ of the glutathione in the substantia nigra is in the oxidized form [10]. This study found that most of the total glutathione in various areas of the human brain is in the form of GSSG [10], whereas other authors described GSSG as $1.2 \%$ or less of total glutathione in autopsy specimens of human brain and in fresh samples of monkey brain [16]. GSSG levels comprising $41-100 \%$ of the total glutathione $[10,11]$ are difficult to explain, since they would point to a very unusual state of the redox couple GSH/ GSSG in the human brain. The factors responsible for the conflicting results need to be elucidated but may include differences in tissue storage, tissue extraction or analytical methods.

The decreased concentration of GSH in the substantia nigra in Parkinson's disease could be the result of neuronal loss. In fact, a positive correlation has been shown between the GSH content and the severity of neuronal depletion in the parkinsonian brain [12]. However, neuronal degeneration does not appear to be the only explanation for the GSH reduction observed. since GSSG was not decreased to the same extent as GSH and the percentage from the total glutathione of GSSG was slightly but not significantly increased (Table I). An elevated level of GSSG could therefore serve as an indicator of oxidative stress $[15,21]$. GSH is synthesized from its constituent amino acids by the sequential action of ATPdependent enzymes, gamma-glutamylcysteine synthetase and GSH synthetase. Amino acid precursors for the synthesis of GSH are glycine, cysteine and glutamate. In addition to the reaction processes of GSH as mentioned above. loss of GSH in the substantia nigra in Parkinson's disease may be the result of a disturbance in the biosynthesis. A decrease in available GSH would reduce the capacity of the neurones to detoxify hydrogen peroxide and increase the risk of free radical formation and lipid peroxidation. Increased membrane lipid peroxidation has indeed been shown in the substantia nigra in Parkinson's disease [5], suggesting that free radicals are generated and contribute to neuronal damage.

Elevated striatal GSSG levels following reserpine administration indicate that increased dopamine turnover is associated with oxidative stress [18]. Raised dopamine turnover in Parkinson's disease [6] increases the risk of peroxidative damage in the vicinity of dopamine-containing neurones. This damage could be exacerbated by L-DOPA therapy although there is no direct evidence of mediation by the drug. The capability of I-deprenyl to delay the progression of early Parkinson's disease [9.20] and to increase life-expectancy when combined with LDOPA [2] may be explained by the present results. LDeprenyl reduced oxidative stress associated with increased turnover of dopamine and limited the accumula-

\section{TABLE I}

PATIFNT CHARACTERISTICS AND LEVELS OF REDUCED AND OXIDIZED GLUTATHIONE IN POST-MORTEM SUBSTANTIA NIGRA

Neurochemical values are the means $(\mu \mathrm{g} / \mathrm{g}$ fresh weight $) \pm$ S.E.M. GSH. reduced glutathione; GSSG, oxidized glutathione; GSSG \%, percentage of oxidized glutathione of the total glutathione

\begin{tabular}{lcc} 
& $\begin{array}{c}\text { Control subjects } \\
(n=4)\end{array}$ & $\begin{array}{c}\text { Parkinson's discase } \\
(n=4)\end{array}$ \\
\hline $\begin{array}{l}\text { Mean age } \\
\text { Age range }\end{array}$ & 72 & 76 \\
Sex & 2683 & 6291 \\
Post-mortem time (h) & $4.3 \pm 0.4$ & $1 \mathrm{~F} .3 \mathrm{M}$ \\
Cell loss and presence of & $\mathrm{No}$ & $4.3 \pm 1.3$ \\
Lewy bodies in midbrain & $92.8 \pm 12.6$ & Yes \\
GSH $(\mu \mathrm{g} / \mathrm{g})$ & $2.3 \pm 0.6$ & $49.3 \pm 4.6$ \\
GSSG $(\mu \mathrm{g} / \mathrm{g})$ & $2.3 \pm 0.3$ & $1.7 \pm 0.5$ \\
GSSG $\%$ & & $3.3 \pm 1.0$ \\
\hline
\end{tabular}

$P<0.05$, Wilcoxon's rank-sum test. 
tion of GSSG in the striatum of rodents [4]. Therapy with L-deprenyl may therefore reduce oxidative stress as a consequence of increased dopamine turnover in the surviving nigrostriatal neurones in Parkinson's disease.

The present results do not allow any definite conclusions on the role of GSH and GSSG in the protection from free radicals in the substantia nigra in Parkinson's disease. Further studies using larger sample sizes are necessary to confirm these observations.

1 Ambani, L.M., van Woert, M.H. and Murphy, S., Brain peroxidase and catalase in Parkinson's disease, Arch. Neurol.. 32 (1975) 114 118.

2 Birkmayer, W., Knoll, J., Riederer, P., Youdim, M.B.H., Hars, V. and Marton, J., Increased life expectancy resulting from addition of L-deprenyl to Madopar treatment in Parkinson's disease: a longterm study, J. Neural. Transm., 64 (1985) 113-127.

3 Cohen, G., The pathobiology of Parkinson's disease: biochemical aspects of dopamine neuron senescence, J. Neural Transm., Suppl. 19 (1983) 89-103.

4 Cohen, G. and Spina, M.B., Deprenyl suppresses the oxidant stress associated with increased dopamine turnover, Ann. Neurol., 26 (1989) 689-690.

5 Dexter, D.T., Carter, C.J., Wells, F.R., Javoy-Agid, F., Agid, Y., Lees, A., Jenner, P. and Marsden, C.D., Basal lipid peroxidation in substantia nigra is increased in Parkinson's disease, J. Neurochem., $52(1989) 381 \cdots 389$

6 Hornykiewicz, O. and Kish, S.J., Biochemical pathophysiology of Parkinson's disease. In M.D. Yahr and K.J. Bergmann (Eds.), Parkinson's Disease, Raven, New York, 1986, pp. 19-34.

7 Kish, S.J., Morito, C. and Hornykiewicz, O., Glutathione peroxidase activity in Parkinson's disease brain. Neurosci. Lett., 58 (1985) 343-346.

8 Marsden, C.D., Neuromelanin and Parkinson's disease, J. Neural Transm., Suppl. 19 (1983) 121-141.

9 Parkinson Study Group, Effect of deprenyl on the progression of disability in early Parkinson's disease, N. Engl. J. Med., 321 (1989) 13641371.

10 Perry, T.L., Godin, D.V. and Hansen, S., Parkinson's disease: a disorder due to nigral glutathione deficiency?, Neurosci. Lett., 33 (1982) 305-310.

11 Perry, T.L., Hansen, S., Berry, K., Mok, C. and Lesk, D., Free amino acids and related compounds in biopsies of human brain, $\mathrm{J}$. Neurochem., 18 (1971) 521-528.
12 Riederer, P., Sofic, E., Rausch, W.D., Schmid1. B., Reynolds, G.P'. Jellinger, K. and Youdim, M.B.H., Transition metals, ferritin, gitutathione, and ascorbic acid in parkinsonian brains. J. Ncurochem.. 52 (1989) 515520

13 Riederer, P., Strolin Benedetti, M., Dostert. P., Sofic. E., Heuschneider, G. and Guffroy, C.. Do glutathione and ascorbic acid play a role in the neurotoxicity of 1-methyl-4-phenyl-1,2.3,6-tetrahydropyridine?, Pharmacol. Toxicol., 60 Suppl. 1 (1987) 39.

14 Rosengren, E.. Linder Eliasson, E. and Carlsson, A., Detection of 5-S-cysteinyldopamine in human brain. J. Neural Transm.. 63 (1985) 247253

15 Sies, H. Hydroperoxides and thiol oxidants in the study of oxidative stress in intact cells and organs. In $\mathrm{H}$. Sies (Ed.). Oxidative Stress, Academic Press, London, 1983, pp. 73-90.

16 Slivka. A.. Spina, M.B. and Cohen, G., Reduced and oxidized glutathione in human and monkey brain, Neurosci. Lett., 74 (1987) $112-118$.

17 Sofic. E., Riederer, P., Burger, R., Gsell, W. and Heuschneider, G., Determination of glutathione, glutathione disulphide, ascorbic acid and dehydroascorbic acid in tissues by reversed-phase liquid chromatography with electrochemical detection, Fresenius J. Anal. Chem., 339 (1991) 258-260.

18 Spina, M.B. and Cohen, G., Dopamine tumover and glutathione oxidation: implications for Parkinson's disease. Proc. Natl. Acad. Sci. USA. 88 (1989) 1398-1400.

19 Strolin Benedetti, M., Dostert, P. and Guffroy. C.. The possible relation of glutathione and MPTP to Parkinson's disease. In S.P. Markey, N. Castagnoli, A.J. Trevor and I.J. Kopin (Eds.), MPTP: A Neurotoxin Producing a Parkinsonian Syndrome, Academic Press, Orlando, 1986, pp. 455460.

20 Tetrud, J.W. and Langston, J.W. The effect of deprenyl (selegiline) on the natural history of Parkinson's disease. Science, 245 (1989) 519-522.

21 White, C.W., Mimmack, R.F. and Repine, J.E., Accumulation of lung tissue oxidized glutathione (GSSG) as a marker of oxidant induced lung injury, Chest (Suppl.), 89 (1986) $111 \cdots 113$.

22 Wilcoxon, F. Individual comparisons by ranking methods, Biometrics. 1 (1945) 80 83.

23 Yong. V.W., Perry, T.L. and Krisman, A.A., Depletion of glutathione in brainstem of mice caused by $\mathbf{N}$-methyl-4-phenyl-1,2,3,6tetrahydropyridine is prevented by antioxidant pretreatment, Neurosci. Lett.. 63 (1986) 56-60. 